\title{
Investigation into the promoter DNA methylation of three genes (CAMK1D, CRY2 and CALM2) in the peripheral blood of patients with type 2 diabetes
}

\author{
JIA CHENG $^{1,2^{*}}$, LINLIN TANG ${ }^{1 *}$, QINGXIAO HONG ${ }^{1}$, HUADAN YE $^{1}, \mathrm{XUTING} \mathrm{XU}^{1}$, LEITING XU ${ }^{1}$, \\ SHIZHONG BU $^{1}$, QINWEN WANG ${ }^{1}$, DONGJUN DAI ${ }^{1}$, DANJIE JIANG ${ }^{1}$ and SHIWEI DUAN ${ }^{1}$
}

\author{
${ }^{1}$ Zhejiang Provincial Key Laboratory of Pathophysiology, School of Medicine, Ningbo University, Ningbo, Zhejiang 315211; \\ ${ }^{2}$ Department of Clinical Medicine, Ningbo Kangning Hospital, Ningbo, Zhejiang 315201, P.R. China
}

Received January 11, 2014; Accepted May 19, 2014

DOI: $10.3892 /$ etm.2014.1766

\begin{abstract}
Promoter DNA methylation may reflect the interaction between genetic backgrounds and environmental factors in the development of metabolic disorders, including type 2 diabetes (T2D). Calcium/calmodulin-dependent protein kinase 1D (CAMK1D), cryptochrome 2 (CRY2) and calmodulin 2 (CALM2) genes have been identified to be associated with a risk of T2D. Therefore, the aim of the present study was to investigate the contribution of promoter DNA methylation of these genes to the risk of T2D. Using bisulfite pyrosequencing technology, the DNA methylation levels of the $\mathrm{CpG}$ dinucleotides within the CAMK1D, CRY2 and CALM2 gene promoters were measured in 48 patients with T2D and 48 age- and gender-matched healthy controls. The results demonstrated that the promoters of these three genes were hypomethylated in the peripheral blood of all the subjects, and DNA methylation of these three genes did not contribute to the risk of T2D.
\end{abstract}

\section{Introduction}

Type 2 diabetes (T2D) is a major public health problem in numerous countries $(1,2)$. Genetic and environmental factors

Correspondence to: Professor Shiwei Duan, Zhejiang Provincial Key Laboratory of Pathophysiology, School of Medicine, Ningbo University, 818 Fenghua Road, Ningbo, Zhejiang 315211, P.R. China E-mail: duanshiwei@nbu.edu.cn

*Contributed equally

Abrreviations: T2D, type 2 diabetes; CAMK1D, calcium/calmodulin-dependent protein kinase 1D; CRY2, cryptochrome 2; CALM2, calmodulin 2; CI, confidence interval; ALT, alanine aminotransferase; SNP, single nucleotide polymorphism; PCR, polymerase chain reaction

Key words: type 2 diabetes, calcium/calmodulin-dependent protein kinase 1D, cryptochrome 2, calmodulin 2, DNA methylation, promoter are hypothesized to contribute to the initiation and development of the disease (3). Genetic association studies based on genome-wide association and meta-analyses have revealed a number of susceptible genetic polymorphisms associated with the pathogenesis of T2D $(2,4-7)$. However, a number of these polymorphisms are not functional variants and are most likely cotransmitted with the causative polymorphisms in large linkage disequilibrium blocks, and do not directly control the expression of T2D susceptibility genes (8). Evaluating the impact of these genetic-environmental interactions and predicting the personal onset risk of developing T2D remains a challenge.

Epigenetic regulation is sensitive to a number of environmental factors and may provide a bridge between the environment and genetic background (1). As a crucial mechanism in epigenetic regulation, DNA methylation regulates gene expression by transferring a methyl group to cytosine nucleotides via DNA methyltransferase (1). Unlike genetic polymorphisms, DNA methylation levels may alter during numerous processes, including development, tissue differentiation and aging (1). In a monozygotic twins study, the older twins showed more distinct changes according to DNA methylation profiles in the epigenome $(9,10)$. Environmental factors, including nutrition and lifestyle, may influence DNA methylation in mammals. For example, the promoter methylation of certain genes was increased in human primary muscle cells due to the exposure of the free fatty acids palmitate (10). A series of genes in primary metabolic processes were reported to show a differential methylation status in skeletal muscle in patients with T2D compared with normal glucose-tolerant individuals (11). Furthermore, dysregulation of promoter methylation has been found in pancreatic islets from patients with T2D (1). Elucidating the association between promoter methylation and the pathogenesis of T2D is a promising field in the research of metabolic disorders $(1,10)$.

The calcium/calmodulin-dependent protein kinase 1D (CAMK1D) gene encodes a member of the $\mathrm{Ca}^{2+} /$ calmodulin-dependent protein kinase 1 subfamily of serine/threonine kinases that plays an important role in the regulation of granulocyte function via the chemokine signaling transduction 
pathway $(12,13)$. Single nucleotide polymorphisms (SNPs) in CAMK1D have been found to be associated with the susceptibility of developing T2D in an east Asian population (14). However, no significant association between the CAMK1D SNP and T2D was found in a study based on European populations (15). Thus, the role of the CAMK1D gene in the pathophysiology of T2D remains unclear (13). The calmodulin 2 (CALM2) gene encodes a member of the human calmodulin family, and polymorphisms in this gene have been associated with dialysis survival in T2D-associated renal disease (16). The cryptochrome 2 (CRY2) gene is a circadian signal gene, whose genetic variation has been associated with T2D and metabolic characteristics $(17,18)$. A recent study investigating monozygotic twins found that the methylation status of CRY2 in subcutaneous adipose tissue differed between the twin with T2D and the healthy twin (18). Further investigations into the association between promoter methylation of the CRY2 gene and T2D in the peripheral blood should be conducted.

Aberrant gene methylation has been identified not only in diseased tissues, but also in human peripheral tissue, including blood lymph cells (8). Detecting the promoter methylation levels of T2D-associated genes in the peripheral blood may be beneficial for the identification of novel diabetic biomarkers with preventative and/or diagnostic values. In the present study, the methylation levels of three candidate genes (CAMK1D, CRY2 and CALM2) in patients with T2D and non-diabetic individuals were investigated to determine the value of this epigenetic marker, with the aim of providing further understanding into the disease etiology of T2D.

\section{Materials and methods}

Subjects. A total of 48 patients with T2D and 48 age- and gender-matched healthy controls were recruited from the Affiliated Hospital of Ningbo University (Ningbo, China). The characteristics of the individuals are shown in Table I. All the individuals were of Han Chinese origin and had lived in the Ningbo area for at least three generations. Patients with T2D were recruited if their plasma glucose levels were $>7.0 \mathrm{mmol} / 1$ at fasting or $>11.1 \mathrm{mmol} / \mathrm{l}$ at $2 \mathrm{~h}$ following glucose load (World Health Organization) (19). Healthy individuals were recruited according to the standard of fasting blood glucose of $<6.1 \mathrm{mmol} / \mathrm{l}$. None of the controls had a family history of T2D in first-degree relatives or had received any medication. Subjects were excluded from the study if they had hypertension, coronary heart disease, renal inadequacy, drug abuse or any other serious diseases. The study was approved by the Ethics Committee of Ningbo University and written informed consent was obtained from all the subjects. Blood samples were collected in $3.2 \%$ citrate sodium-treated tubes and stored at $-80^{\circ} \mathrm{C}$ for DNA extraction.

Phenotype collection. Blood samples were collected from the antecubital vein into vacutainer tubes containing ethylene diamine tetraacetic acid following fasting for $12 \mathrm{~h}$ overnight. Plasma levels of cholesterol, triglyceride, alanine aminotransferase (ALT), uric acid and glucose concentrations were enzymatically measured using the CX7 Analyzer (Beckman Coulter, Inc., Fullerton, CA, USA).
DNA methylation assay. Human genomic DNA was isolated from peripheral blood samples using a nucleic acid extraction automatic analyzer (Lab-Aid 820; Xiamen, Fujian, China). DNA was quantified using the PicoGreen double strand DNA Quantification kit (Molecular Probes, Inc., Eugene, OR, USA). Bisulfite pyrosequencing technology was used to determine the $\mathrm{CpG}$ dinucleotide methylation levels of fragments within the promoters of the CAMK1D, CRY2 and CALM2 genes (Fig. 1). The pyrosequencing assays involved sodium bisulfite DNA conversion chemistry, polymerase chain reaction (PCR) amplification and sequencing by synthesis assay of the target sequence. Sodium bisulfite preferentially deaminates unmethylated cytosine residues to thymines (following PCR amplification), while methyl-cytosines remain unmodified. PCR primers were selected using PyroMark Assay Design software v2.0.1.15 and the amplification primers for the CAMK1D, CRY2 and CALM2 gene promoters are shown in Table II.

Statistical analysis. Pearson's $\chi^{2}$ test was used to compare the categorical variables, while mean group differences for continuous variables were compared using the Student's t-test. Pearson's correlation analysis was applied to determine the associations between the methylation status and metabolic features of the subjects. $\mathrm{P}<0.05$ was considered to indicate a statistically significant difference. All statistical analyses were performed using PASW Statistics 18.0 software (SPSS, Inc., Chicago, IL, USA).

\section{Results}

Low methylation levels within the CAMK1D, CRY2 and CALM 2 promoters. A total of 48 patients with T2D and 48 age- and gender-matched controls were recruited for the association study, and the methylation levels of three genes (CAMK1D, CRY2 and CALM2) were investigated. In total, four $\mathrm{CpG}$ dinucleotides within the CALM2 gene promoter, five $\mathrm{CpG}$ dinucleotides within the CRY2 gene promoter and nine $\mathrm{CpG}$ dinucleotides within the CAMK1D gene promoter were identified. The correlations between the DNA methylation levels among all the CpGs are shown in Fig. 1 and the characteristics of the subjects are shown in Table I. As shown in Table I, the promoters of the three genes in the peripheral blood exhibited low methylation levels for all the subjects. The DNA methylation information of all the CpGs within the CAMK1D, CRY2 and CALM2 promoters is shown in Fig. 2.

Statistical analysis of clinical phenotypes. No statistically significant differences in clinical phenotypes, including age, body mass index, total cholesterol and uric acid, were observed between the T2D and controls patients (Table I; P>0.05). However, the glucose level in patients with T2D was significantly higher compared with the control patients (Table I; $\mathrm{P}<0.001$ ), and a similar result was observed in the breakdown analysis by gender. The levels of ALT and total triglycerides were higher in patients with T2D (Table I; $\mathrm{P}=0.028$ and $\mathrm{P}=0.034$, respectively). However, in the breakdown analysis by gender, no differential level of total triglycerides was observed. Notably, higher levels of ALT in female patients with T2D were observed (Table III; $\mathrm{P}=0.019$ ). 
Table I. Characteristics of the subjects $(n=96)$.

\begin{tabular}{|c|c|c|c|c|c|}
\hline Characteristics & Value & Range & $\mathrm{T} 2 \mathrm{D}$ & Controls & P-value \\
\hline Age (years) & $59.2 \pm 7.5$ & $35-69$ & $59.2 \pm 7.5$ & $59.2 \pm 7.5$ & 1.000 \\
\hline Gender (M/F) & $48 / 48$ & - & - & - & - \\
\hline BMI $\left(\mathrm{kg} / \mathrm{m}^{2}\right)$ & $23.71 \pm 3.28$ & $17.15-42.96$ & $24.17 \pm 4.18$ & $23.18 \pm 1.64$ & 0.146 \\
\hline Total cholesterol (mmol/l) & $5.19 \pm 0.96$ & $2.95-7.90$ & $5.34 \pm 0.83$ & $5.05 \pm 1.06$ & 0.140 \\
\hline Total triglycerides $(\mathrm{mmol} / \mathrm{l})$ & $1.60 \pm 1.36$ & $0.40-9.92$ & $1.90 \pm 1.69$ & $1.31 \pm 0.82$ & 0.034 \\
\hline Glucose (mmol/l) & $6.76 \pm 2.65$ & $4.38-22.84$ & $8.31 \pm 2.91$ & $5.22 \pm 0.92$ & 0.000 \\
\hline ALT (IU/l) & $21.5 \pm 15.9$ & $5.0-99.0$ & $25.1 \pm 18.5$ & $18.0 \pm 12.1$ & 0.028 \\
\hline Uric acid $(\mu \mathrm{mol} / \mathrm{l})$ & $294.9 \pm 81.1$ & $132.0-531.0$ & $289.3 \pm 70.5$ & $300.6 \pm 90.9$ & 0.499 \\
\hline \multicolumn{6}{|l|}{ CALM2 methylation (\%) } \\
\hline CpG1 & $0.96 \pm 0.38$ & $0-2$ & $1.02 \pm 0.40$ & $0.88 \pm 0.33$ & 0.099 \\
\hline CpG2 & $2.01 \pm 0.50$ & $0-3$ & $2.18 \pm 0.39$ & $1.79 \pm 0.55$ & 0.001 \\
\hline CpG3 & $0.92 \pm 0.48$ & $0-2$ & $1.00 \pm 0.53$ & $0.82 \pm 0.39$ & 0.087 \\
\hline CpG4 & $1.40 \pm 0.86$ & $0-5$ & $1.11 \pm 0.58$ & $1.79 \pm 1.02$ & 0.001 \\
\hline \multicolumn{6}{|l|}{ CRY2 methylation (\%) } \\
\hline CpG1 & $1.25 \pm 0.53$ & $0-2$ & $1.13 \pm 0.41$ & $1.44 \pm 0.65$ & 0.022 \\
\hline CpG2 & $0.80 \pm 0.41$ & $0-1$ & $0.79 \pm 0.41$ & $0.80 \pm 0.41$ & 0.961 \\
\hline CpG3 & $1.61 \pm 0.63$ & $0-3$ & $1.64 \pm 0.63$ & $1.56 \pm 0.65$ & 0.621 \\
\hline CpG4 & $0.98 \pm 0.42$ & $0-3$ & $1.05 \pm 0.39$ & $0.88 \pm 0.44$ & 0.110 \\
\hline CpG5 & $1.14 \pm 3.32$ & $0-27$ & $0.64 \pm 0.54$ & $1.92 \pm 5.24$ & 0.134 \\
\hline \multicolumn{6}{|l|}{ CAMK1D methylation (\%) } \\
\hline CpG1 & $1.04 \pm 0.29$ & $0-3$ & $1.04 \pm 0.20$ & $1.04 \pm 0.36$ & 1.000 \\
\hline CpG2 & $0.75 \pm 0.48$ & $0-2$ & $0.90 \pm 0.31$ & $0.60 \pm 0.57$ & 0.003 \\
\hline CpG3 & $1.53 \pm 0.54$ & $1-3$ & $1.75 \pm 0.48$ & $1.31 \pm 0.51$ & 0.000 \\
\hline CpG4 & $1.04 \pm 0.29$ & $0-2$ & $1.10 \pm 0.31$ & $0.98 \pm 0.25$ & 0.032 \\
\hline CpG5 & $1.02 \pm 0.20$ & $0-2$ & $1.04 \pm 0.20$ & $1.00 \pm 0.21$ & 0.320 \\
\hline CpG6 & $0.96 \pm 0.32$ & $0-2$ & $1.02 \pm 0.14$ & $0.90 \pm 0.43$ & 0.057 \\
\hline CpG7 & $1.04 \pm 0.29$ & $0-2$ & $1.06 \pm 0.38$ & $1.02 \pm 0.14$ & 0.480 \\
\hline CpG8 & $0.92 \pm 0.45$ & $0-3$ & $1.02 \pm 0.25$ & $0.81 \pm 0.57$ & 0.023 \\
\hline CpG9 & $0.82 \pm 0.54$ & $0-3$ & $1.00 \pm 0.41$ & $0.65 \pm 0.60$ & 0.001 \\
\hline
\end{tabular}

Results are expressed as the mean \pm standard error. ${ }^{a} \mathrm{n}=86$ (46 patients with T2D vs. 40 control patients). T2D, type 2 diabetes; BMI, body mass index; ALT, alanine aminotransferase; CALM2, calmodulin 2; CRY2, cryptochrome 2; CAMK1D, calcium/calmodulin-dependent protein kinase 1D; M, male; F, female.

\section{Discussion}

Epigenetic regulation is involved in numerous types of human diseases, and epigenetic biomarkers have been reported to be associated with pathological processes, of which a number have potential value as laboratory diagnostic tools (20-23). DNA methylation, as one of the most studied epigenetic regulatory mechanisms, is essential in the interaction between genetic and environmental factors $(10,20)$. DNA methylation usually occurs in the $\mathrm{CpG}$ islands of functional gene promoter regions (20), and this type of epigenetic modification was originally considered to be stable in vitro and function as a tissue-specific feature in disease $(10,24,25)$. A number of disease-associated DNA methylation variations have been identified in a diverse range of tissues, including the peripheral blood $(8,10,20,26)$.

T2D is a major metabolic disease, which physiological and pathological states may be regulated by epigenetic modifications that control gene expression (10). Hundreds of differential DNA methylation CpGs of genes promoters have been identified in genomic DNA isolated from pancreatic islets using Infinium methylation assay technology (1). These disease-specific methylation signatures also exist in human accessible peripheral tissues (8). A prospective study found that significant hypomethylation of young individuals was associated with a higher risk of developing T2D in later life (8). In the present study, the DNA promoter methylation levels of three T2D candidate genes (CALM2, CRY2 and CAMK1D) in the peripheral blood were investigated, providing further information regarding DNA promoter methylation regulation in T2D.

Low levels of DNA promoter methylation were identified in the three genes in the healthy subjects and patients with T2D (Table I). The majority of the methylation correlation coefficients of the CpGs within these genes were also not 
Table II. Primers for the promoter CpG methylation analysis.

Gene Sequence

\section{CALM2}

Forward primer

Reverse primer

Sequencing primer

CRY2

Forward primer

Reverse primer

Sequencing primer

CAMK1D

Forward primer

Reverse primer

Sequencing primer

\section{5'-AGGAGGAGTTGTTGGAGAATATGA-3' \\ 5'-biotin- ACTACCCCCCTAACCCCTCT-3' \\ 5'-GTTTTGAGTGTTTAGGTAAGG-3'}

\author{
5'-GGGGTGGTTGGAGTAGTTTGG-3' \\ 5'-biotin-AATCCCCTCACCTCCATC-3' \\ 5'-GGAGTAGTTTGGATAGTTA-3'
}

5'-GGAGGTAAGAAAGTAGTAGAAAGTGA-3'
5'-biotin-CCTCCTCTACAATTTCCTCTT-3'
5'-GAGTTAGGGAGGGAT-3'

CALM2, calmodulin 2; CRY2, cryptochrome 2; CAMK1D, calcium/calmodulin-dependent protein kinase 1D.

A

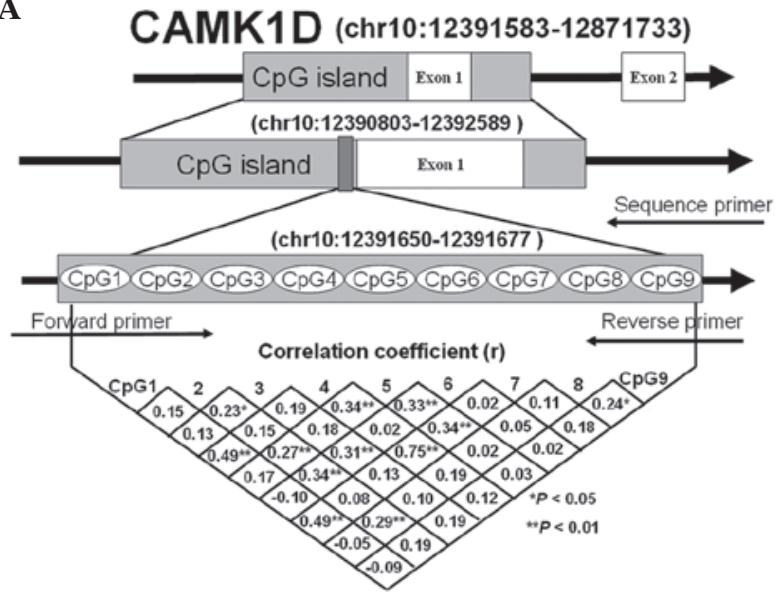

B CALM2 (chr: $47,383,091-47,407,870)$

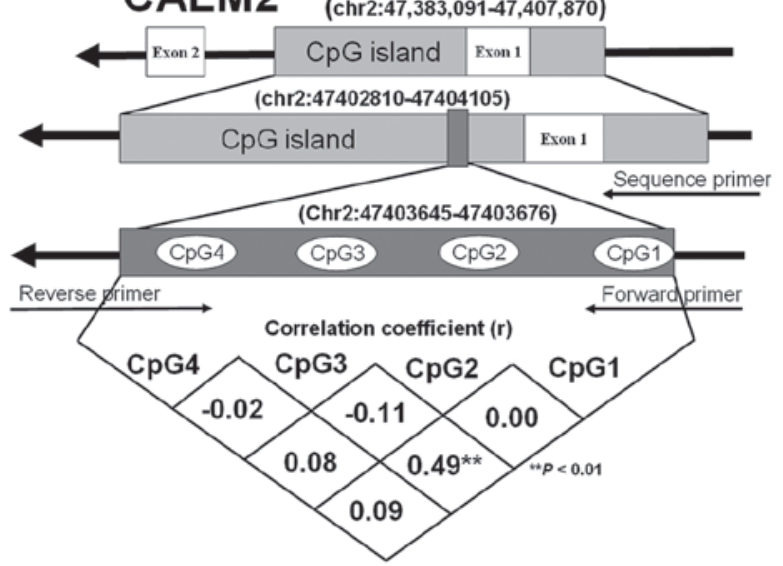

C

CRY2 (chr11:45,868,957-45,904,799)

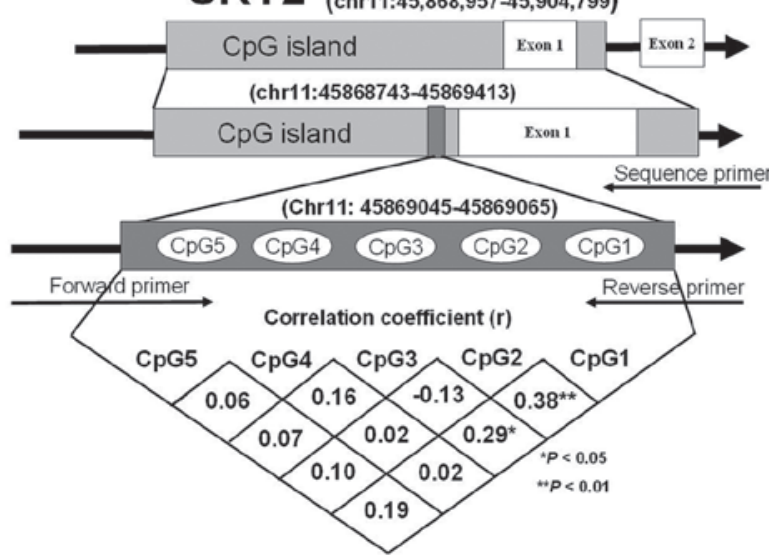

Figure 1. CpG islands within the CAMK1D, CRY2 and CALM2 promoters. The diagrams indicate the correlation coefficients, and (A) nine CpG dinucleotides within CAMK1D, (B) four CpG dinucleotides within CALM2 and (C) five CpG dinucleotides within CRY2. ${ }^{*} \mathrm{P}<0.05,{ }^{* *} \mathrm{P}<0.01$. P-values were calculated by Pearson test which analyzed the correlation of the methylation levels among the tested CpGs. CALM2, calmodulin 2; CRY2, cryptochrome 2; CAMK1D, calcium/calmodulin-dependent protein kinase 1D.

significant (Fig. 1). Two reasons may explain these observations. In epigenetics, not all candidate genes are directly regulated by DNA methylation, with other epigenetic factors, including non-coding RNA and histone variants, also involved in the regulation of gene expression $(20,23)$. Since $\mathrm{T} 2 \mathrm{D}$ is a polygenic disease, the low DNA promoter meth- 
Table III. Characteristics of the subjects by gender.

\begin{tabular}{lccc}
\hline Characteristics & T2D & Controls \\
\hline Males $(\mathrm{n}=48)$ & & & \\
Age $($ years) & $59.1 \pm 8.7$ & $59.1 \pm 8.7$ & $23.10 \pm 1.21$ \\
BMI $\left(\mathrm{kg} / \mathrm{m}^{2}\right)^{\mathrm{a}}$ & $24.92 \pm 5.17$ & $4.86 \pm 1.11$ & 0.124 \\
Total cholesterol $(\mathrm{mmol} / \mathrm{l})$ & $5.06 \pm 0.74$ & $1.38 \pm 0.90$ & 0.464 \\
Total triglycerides $(\mathrm{mmol} / \mathrm{l})$ & $1.81 \pm 1.56$ & $4.94 \pm 0.34$ & 0.254 \\
Glucose $(\mathrm{mmol} / \mathrm{l})$ & $8.59 \pm 3.49$ & $21.1 \pm 15.9$ & 0.000 \\
ALT $(\mathrm{IU} / \mathrm{l})$ & $30.4 \pm 23.8$ & $346.5 \pm 82.7$ \\
Uric acid $(\mu \mathrm{mol} / \mathrm{l})$ & $304.7 \pm 70.6$ & & 0.119 \\
Females $(\mathrm{n}=48)$ & & $59.4 \pm 6.4$ & 0.066 \\
Age $($ years $)$ & $59.4 \pm 6.4$ & $23.25 \pm 1.93$ \\
BMI $\left(\mathrm{kg} / \mathrm{m}^{2}\right)^{\mathrm{b}}$ & $23.49 \pm 2.97$ & $5.24 \pm 1.00$ \\
Total cholesterol $(\mathrm{mmol} / \mathrm{l})$ & $5.62 \pm 0.83$ & $1.23 \pm 0.75$ \\
Total triglycerides $(\mathrm{mmol} / \mathrm{l})$ & $1.98 \pm 1.85$ & $5.49 \pm 1.20$ \\
Glucose $(\mathrm{mmol} / \mathrm{l})$ & $8.04 \pm 2.22$ & $14.8 \pm 4.8$ \\
ALT $(\mathrm{IU} / \mathrm{l})$ & $19.8 \pm 8.6$ & $254.6 \pm 75.0$ \\
Uric acid $(\mu \mathrm{mol} / \mathrm{l})$ & $273.9 \pm 68.3$ & 0.747 \\
\hline
\end{tabular}

${ }^{a} \mathrm{n}=39$ ( 22 patients with T2D vs. 17 control patients); ${ }^{b} \mathrm{n}=47$ (24 patients with T2D vs. 23 control patients). T2D, type 2 diabetes; BMI, body mass index; ALT, alanine aminotransferase; SE, standard error.
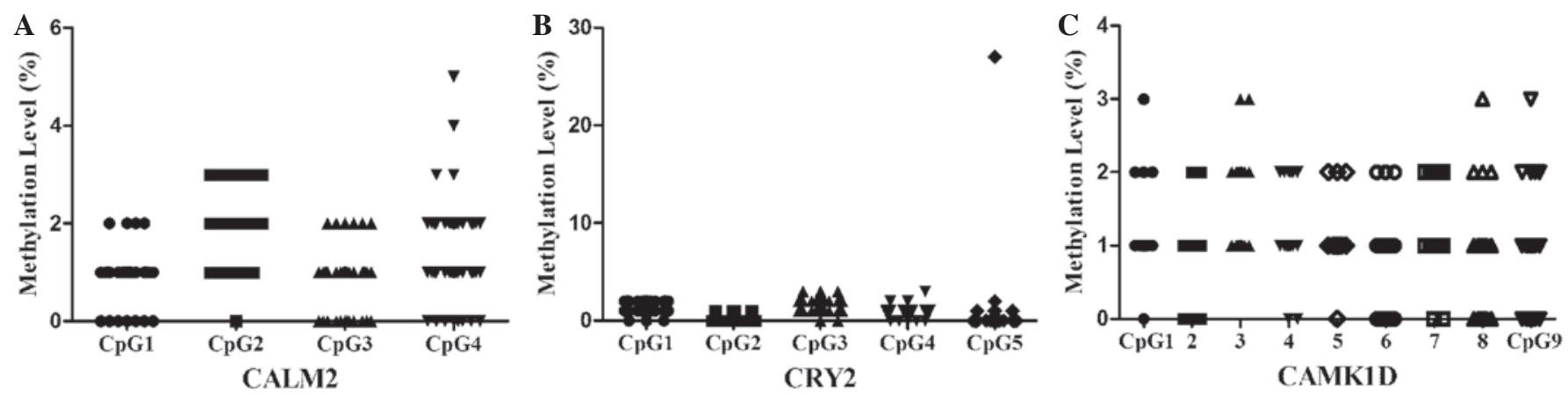

Figure 2. DNA methylation of all the CpGs within the CAMK1D, CRY2 and CALM2 promoters. Methylation levels of each dinucleotide of the (A) four CpGs within CALM2, (B) five CpGs within CRY2 and (C) nine CpGs within CAMK1D. CALM2, calmodulin 2; CRY2, Cryptochrome 2; CAMK1D, calcium/calmodulin-dependent protein kinase 1D.

ylation levels of CALM2, CRY2 and CAMK1D genes may indicate that DNA promoter methylation has no direct effect on gene function. In addition, the methylation status was only investigated in the peripheral blood, and DNA promoter methylation modification has been demonstrated to exhibit a tissue-specific methylation pattern (25). The insulin-2 gene, an additional candidate gene for diabetes, was found to be unmethylated in $\beta$ cells, but methylated in other tissues (25). The identification of DNA methylation profiling in pancreatic islets revealed that differences in the methylation status of certain candidate genes between T2D and non-diabetic patients were not present in blood cells (1). The two aforementioned reasons may explain the low DNA methylation levels of CALM2, CRY2 and CAMK1D observed in the present study.

In conclusion, low methylation levels of CALM2, CRY2 and CAMK1D were observed in the peripheral blood of the healthy controls and T2D patients. These observations indicate that DNA methylation may not be the primary regulatory mechanism of CALM2, CRY2 and CAMK1D in T2D, and these methylation loci may not be regarded as biomarkers for T2D. The present study provides further information with regard to the epigenetic mechanisms and may provide reference value in genetic-based pharmacological development for future T2D treatments.

\section{Acknowledgements}

The study was supported by grants from the National Natural Science Foundation of China (nos. 31100919 and 81371469), the Natural Science Foundation of Zhejiang Province (no. LR13H020003) and the K.C. Wong Magna Fund in Ningbo University, Ningbo Social Development Research Projects (no. 2012C50032). 


\section{References}

1. Volkmar M, Dedeurwaerder S, Cunha DA, et al: DNA methylation profiling identifies epigenetic dysregulation in pancreatic islets from type 2 diabetic patients. EMBO J 31: 1405-1426, 2012

2. Omori S, Tanaka Y, Takahashi A, et al: Association of CDKAL1, IGF2BP2, CDKN2A/B, HHEX, SLC30A8, and KCNJ11 with susceptibility to type 2 diabetes in a Japanese population. Diabetes 57: 791-795, 2008.

3. Gu T, Horová E, Möllsten A, et al: IGF2BP2 and IGF2 genetic effects in diabetes and diabetic nephropathy. J Diabetes Complications 26: 393-398, 2012.

4. Kwak SH, Kim SH, Cho YM, et al: A genome-wide association study of gestational diabetes mellitus in Korean women. Diabetes 61: 531-541, 2012

5. Grarup N, Rose CS, Andersson EA, et al: Studies of association of variants near the HHEX, CDKN2A/B, and IGF2BP2 genes with type 2 diabetes and impaired insulin release in 10,705 Danish subjects: validation and extension of genome-wide association studies. Diabetes 56: 3105-3111, 2007.

6. Jia H, Yu L, Jiang Z and Ji Q: Association between IGF2BP2 rs4402960 polymorphism and risk of type 2 diabetes mellitus: a meta-analysis. Arch Med Res 42: 361-367, 2011.

7. Wu J, Wu J,Zhou Y, et al: Quantitative assessment of the variation in IGF2BP2 gene and type 2 diabetes risk. Acta Diabetol 49 (Suppl 1): S87-S97, 2012.

8. Toperoff G, Aran D, Kark JD, et al: Genome-wide survey reveals predisposing diabetes type 2-related DNA methylation variations in human peripheral blood. Hum Mol Genet 21: 371-383, 2012.

9. Fraga MF, Ballestar E, Paz MF, et al: Epigenetic differences arise during the lifetime of monozygotic twins. Proc Natl Acad Sci USA 102: 10604-10609, 2005.

10. Barres R and Zierath JR: DNA methylation in metabolic disorders. Am J Clin Nutr 93: 897S-900S, 2011.

11. Barrès R, Osler ME, Yan J, et al: Non-CpG methylation of the PGC-1alpha promoter through DNMT3B controls mitochondrial density. Cell Metab 10: 189-198, 2009.

12. Verploegen S, Ulfman L, van Deutekom HW, et al: Characterization of the role of CaMKI-like kinase (CKLiK) in human granulocyte function. Blood 106: 1076-1083, 2005.

13. Shu XO, Long J, Cai Q, et al: Identification of new genetic risk variants for type 2 diabetes. PLoS Genet 6: e1001127, 2010.

14. Imamura $\mathrm{M}$, Iwata $\mathrm{M}$, Maegawa $\mathrm{H}$, et al: Genetic variants at CDC123/CAMK1D and SPRY2 are associated with susceptibility to type 2 diabetes in the Japanese population. Diabetologia 54 3071-3077, 2011.
15. Schleinitz D, Tönjes A, Böttcher Y, et al: Lack of significant effects of the type 2 diabetes susceptibility loci JAZF1, CDC123/CAMK1D, NOTCH2, ADAMTS9, THADA, and TSPAN8/LGR5 on diabetes and quantitative metabolic traits. Horm Metab Res 42: 14-22, 2010.

16. Murea M, Lu L, Ma L, et al: Genome-wide association scan for survival on dialysis in African-Americans with type 2 diabetes. Am J Nephrol 33: 502-509, 2011.

17. Dupuis J, Langenberg C, Prokopenko I, et al: New genetic loci implicated in fasting glucose homeostasis and their impact on type 2 diabetes risk. Nat Genet 42: 105-116, 2010.

18. Ribel-Madsen R, Fraga MF, Jacobsen S, et al: Genome-wide analysis of DNA methylation differences in muscle and fat from monozygotic twins discordant for type 2 diabetes. PLoS One 7: e51302, 2012.

19. Alberti KG and Zimmet PZ: Definition, diagnosis and classification of diabetes mellitus and its complications. Part 1: diagnosis and classification of diabetes mellitus provisional report of a WHO consultation. Diabet Med 15: 539-553, 1998.

20. García-Giménez JL, Sanchis-Gomar F, Lippi G, et al: Epigenetic biomarkers: A new perspective in laboratory diagnostics. Clin Chim Acta 413: 1576-1582, 2012.

21. Zampetaki A, Kiechl S, Drozdov I, et al: Plasma microRNA profiling reveals loss of endothelial miR-126 and other microRNAs in type 2 diabetes. Circ Res 107: 810-817, 2010.

22. Zhao C, Dong J, Jiang T, et al: Early second-trimester serum miRNA profiling predicts gestational diabetes mellitus. PLoS One 6: e23925, 2011.

23. Dehwah MA, Xu A and Huang Q: MicroRNAs and type 2 diabetes/obesity. J Genet Genomics 39: 11-18, 2012.

24. Poy MN, Eliasson L, Krutzfeldt J, et al: A pancreatic islet-specific microRNA regulates insulin secretion. Nature 432: 226-230, 2004.

25. Husseiny MI, Kuroda A, Kaye AN, Nair I, Kandeel F and Ferreri K: Development of a quantitative methylation-specific polymerase chain reaction method for monitoring beta cell death in type 1 diabetes. PLoS One 7: e47942, 2012.

26. Kong L, Zhu J, Han W, et al: Significance of serum microRNAs in pre-diabetes and newly diagnosed type 2 diabetes: a clinical study. Acta Diabetol 48: 61-69, 2011. 